\title{
Propiedades psicométricas de la versión española de la escala de resiliencia de 10 ítems de Connor-Davidson (CD-RISC 10) en una muestra de desempleados
}

\section{Psychometric properties of Spanish version of 10-item Connor-Davidson resilience scale (10-item CD-RISC) in an unemployed sample}

\author{
María Magdalena Fernández Valera, Mariano Meseguer de Pedro, María Isabel Soler Sánchez \\ Universidad de Murcia, España
}

(Rec.: abril de 2017 - Acept.: agosto de 2017)

\begin{abstract}
Resumen
El objetivo del estudio es analizar las propiedades psicométricas de la escala de resiliencia de Connor y Davidson (2003) en su versión reducida de 10 ítems (CD-RISC 10) desarrollada por Campbell-Stills y Stein (2007) y validada al español por Notario-Pacheco et al. (2011) en una muestra de 226 desempleados. Se llevó a cabo un análisis factorial confirmatorio (AFC) que corroboró el modelo unifactorial. Siguiendo las aportaciones teóricas, se propusieron las variables de salud psicológica, síntomas psicosomáticos y autoeficacia para analizar la validez de criterio. Las correlaciones entre las variables fueron estadísticamente significativas y siguieron la dirección teórica esperada. Se concluye que la versión española de 10 ítems de la CD-RISC de Connor y Davidson (2003) presenta adecuadas propiedades psicométricas para estimar la resiliencia en desempleados españoles.
\end{abstract}

Palabras clave: CD-RISC, desempleados, resiliencia.

\begin{abstract}
The aim of this study was to analyze the psychometric properties of the Spanish adaptation of the scale of resilience CD-RISC by Connor and Davidson (2003), in its 10-item version developed by Campbell-Stills and Stein (2007), in a sample of 226 unemployed. We carried out a confirmatory factor analysis (CFA) which corroborated the univariate model. Following the theoretical contributions, we proposed psychological health, psychosomatic symptoms and self-efficacy as variables to analyze the criterion validity. The correlations between the variables were statistically significant and followed the expected theoretical direction. We concluded that the Spanish 10-item version of the CDRISC by Connor and Davidson (2003) presents adequate psychometric properties to estimate resilience in Spanish unemployed.
\end{abstract}

Keywords: CD-RISC, unemployed, resilience.

Correspondencia: María Magdalena Fernández Valera. Departamento de Psiquiatría y Psicología Social. Facultad de Ciencias del Trabajo. Universidad de Murcia. España. Correo electrónico: mariamagdalena.fernandez@um.es 


\section{Introducción}

Desde el comienzo de la última crisis financiera mundial, iniciada a mediados de la pasada década, el desempleo ha llegado a ser considerado como uno de los principales problemas económicos y sociales en diversos países. La situación de desempleo es un acontecimiento estresante para la persona que se traduce en una experiencia psicológica negativa (McKee-Ryan, Song, Wanberg \& Kinicki, 2005; Paul \& Moser, 2009). Si comparamos a los desempleados con las personas que tienen un trabajo, el primer grupo manifiesta una mayor sintomatología depresiva y ansiógena (Burgard, Brand \& House, 2007; Ford et al., 2010), y, por ende, menor calidad de vida (Del Pozo, Ruiz, Pardo \& San Martín, 2002). Por otro lado, hay otros estudios que confirman que aquellas personas que obtienen un puesto de trabajo consiguen una mejora de su bienestar psicológico y, frecuentemente, de la sintomatología asociada a la salud, en términos generales (Beland, Birch \& Stoddart, 2002; Ferrie et al., 2001; Moyano-Díaz, Gutiérrez, Zúñiga \& Cornejo-Troncoso, 2013).

Teniendo en cuenta que la resiliencia se podría definir como la capacidad de sobreponerse a la adversidad, recuperarse y salir fortalecido con éxito (Csikszentmihalyi, 1999; Grotberg, 1995), nos preguntamos si esta capacidad podría resultar útil en el proceso de afrontamiento de la situación de desempleo. Previo a ello necesitamos estimar el nivel de resiliencia de las personas desempleadas. Sin embargo, ante la escasez de instrumentos de medida de la resiliencia sobre población en situación de desempleo, se considera necesaria la validación de un instrumento que cuente con esas características. Para ello se eligió la escala de estimación de la resiliencia de Connor y Davidson (2003), puesto que ha mostrado excelentes propiedades psicométricas a la hora de estimar la resiliencia en adultos, en su versión reducida de 10 ítems desarrollada por Campbell-Stills y Stein (2007) y validada al español por Notario-Pacheco et al. (2011).

\section{Resiliencia}

La resiliencia se entiende como un proceso dinámico que comprende la adaptación positiva dentro de un contexto significativamente adverso (Luthar, Cicchetti \& Becker, 2000). Por tanto, el concepto de resiliencia tiene que ver con la habilidad de recuperarse de eventos negativos (Becoña, 2006). Para que se suscite esta capacidad, es necesaria la exposición de una persona a situaciones adversas y de riesgo (Fernández-Lansas \& Crespo, 2011; Luthar et al., 2000; Masten, Cutuli, Herbers \& Reed, 2009). El proceso de resiliencia debe incluir determinantes y efectos (Windle, Bennet \& Noyes, 2011). Los primeros, entendidos como aquellos estresores o condiciones ambientales que incrementan la probabilidad de que un individuo tenga resultados negativos en áreas particulares como la salud física, la salud mental, el resultado académico o laboral y el ajuste social. Los efectos son el resultado de la interacción entre los determinantes y la resiliencia, y con frecuencia se suele entender como una adaptación positiva (Luthar et al., 2000; Fergus \& Zimmerman, 2005). En suma, la resiliencia se contempla como una adaptación positiva de la persona a ambientes estresantes y promotora de comportamientos saludables (Baek, Lee, Joo, Lee \& Choi, 2010; Grotberg, 2002; Luthans, 2002; Masten \& Reed, 2002) con capacidad de modificar los efectos en la salud de las personas que se enfrentan a situaciones adversas (Herrman et al., 2011; Luthar \& Becker,
2002; Luthans, Vogelgesang \& Lester, 2006; Pipe et al., 2012; Richardson, 2002).

\section{Resiliencia y desempleo}

Aunque la investigación hasta la fecha ha propiciado un amplio desarrollo del concepto de resiliencia en niños, adolescentes y personas mayores, las investigación sobre esta variable en adultos no ha recibido la misma atención (Moorhouse \& Caltabiano, 2007). El desempleo está considerado como una de las diez experiencias vitales más traumáticas (Hanish, 1999), y como tal, se entiende como una de las principales fuentes de adversidad potenciales en la edad adulta.

Sin embargo, como se acaba de exponer, a pesar de que el desempleo es un acontecimiento altamente estresante, son escasos los estudios que relacionan la resiliencia con la situación de desempleo. El estudio de Moorhouse y Caltabiano (2007) destaca por ofrecer una posible explicación teórica a la relación entre ambas variables. Estos autores examinan el papel de la resiliencia en adultos que se encuentran en situación de desempleo y establecen que esta variable puede proteger a los desempleados de los efectos adversos de la pérdida del trabajo. En concreto, sus resultados muestran que la resiliencia ayuda a afrontar la situación de desempleo a la vez que reduce la probabilidad de síntomas depresivos derivados de la exposición repetida al rechazo y la persistencia propios de la búsqueda de empleo. Encontraron que las personas desempleadas con mayores niveles de resiliencia indicaron menos sintomatología depresiva que aquellos con niveles más bajos, aun cuando su tiempo de búsqueda de empleo era mayor.

Para justificar la aplicación de este recurso psicológico al ámbito del desempleo, argumentan, siguiendo a las teorías desarrolladas sobre la resiliencia (Masten \& Reed, 2002; Rutter, 1993), que a la hora de hacer frente a las adversidades son importantes tres tipos de factores: factores de riesgo, factores de protección y el resultado de conjugar los dos anteriores. Los factores de riesgo son aquellos que incrementan la posibilidad de que se produzca un resultado negativo, en este caso podrían ser: el hecho de contar habilidades pobres de búsqueda de empleo, la necesidad financiera o experimentar largos periodos de desempleo. Por el contrario, los factores de protección son aquellos que median la respuesta individual a la adversidad. De este modo, la experiencia de desempleo puede ser comparable a un trauma (Mol et al., 2005), mientras que la resiliencia puede ser un recurso psicológico personal importante para afrontar la situación de desempleo y reducir sus efectos negativos sobre otras variables como la salud o el proceso de búsqueda de empleo (Moorhouse \& Caltabiano, 2007).

Entre las demás investigaciones que han aplicado la resiliencia al ámbito del desempleo destacan las siguientes: Ślebarska, Moser y Gunnesch-Luca (2009) que analizan si el optimismo y la resiliencia están relacionados con las conductas de búsqueda de empleo. Sus resultados muestran que estos recursos personales median la relación entre el apoyo social y las conductas de búsqueda de empleo. Por su parte, Sweet y Moen (2012) muestran que la preparación para la búsqueda de empleo está relacionada con un aumento de la resiliencia. Taormina (2015) ofrece un enfoque del concepto de resiliencia encuadrado en el movimiento de la psicología positiva. Uno de los resultados más destacables de su estudio confirma que 
los trabajadores tienen puntuaciones más altas en resiliencia que los desempleados. Ello está en consonancia con los postulados de la teoría de la indefensión aprendida o la teoría de la atribución. Tal y como proponen Vansteenkiste, Lens, De Witte y Feather (2005), es posible que el hecho de perder el trabajo pueda influir de manera importante sobre la capacidad de construir y desarrollar la resiliencia en las personas en situación de desempleo. Por último, Lim, Chen y Tan (2013) argumentan que mayores niveles de resiliencia (entendida como un componente del constructo de capital psicológico) permiten a los desempleados comprometerse en los procesos de búsqueda de empleo con una actitud más positiva y, por tanto, perseverar más en la misma, derivando en menores niveles de fatiga. A su vez, exponen que teniendo en cuenta que la búsqueda de empleo se caracteriza por lidiar con la frustración resultante de rechazos repetidos, el hecho de tener menores niveles de resiliencia puede significar que los buscadores de empleo desistan en la búsqueda de empleo, experimenten mayores niveles de fatiga y vean reducida su motivación para buscar un trabajo.

\section{Escala de resiliencia de Connor y Davidson (2003)}

A pesar de contar con numerosas escalas de medida de la resiliencia (Baruth \& Carroll, 2002; González-Arratia, Valdez \& González, 2011; Grotberg, 1995; Wagnild \& Young, 1993), la mayoría no ha conseguido una aceptación unánime, debido, entre otros factores, a la dificultad de extrapolación a grupos de población diferentes. Una de las escalas que más aprobación ha alcanzado en el ámbito científico ha sido la Escala de Resiliencia de Connor y Davidson (CD-RISC; 2003). Son numerosos los autores que la han aplicado en sus investigaciones (Ahem, Kiehl, Sole \& Byers, 2006; Baek et al., 2010; Campbell-Stills \& Stein, 2007; Gillespie, Chaboyerb \& Wallis, 2009; Liu, Fairweather-Schdmidt, Burns \& Roberts, 2015; Lopes \& Martins, 2011; Notario-Pacheco et al., 2011; Soler, Meseguer \& García-Izquierdo, 2016; Sutherland, Cook, Stetina \& Hernández, 2009; Windle et al., 2011).

Esta escala tiene como objetivo estimar el nivel de resiliencia de una persona. Está compuesta por 25 ítems. Los análisis preliminares de esta escala se han desarrollado sobre población general, pacientes de atención primaria así como muestras conformadas por población clínica. Éstos apoyan la consistencia interna de la escala, su fiabilidad test-retest y su validez convergente y divergente. Investigaciones posteriores confirman las excelentes propiedades psicométricas de esta escala para población adulta (por ejemplo, García-Izquierdo, Ramos-Villagrasa \& García-Izquierdo, 2009; Lamond et al., 2008; Liu et al., 2015; Méndez et al., 2015; Sutherland et al., 2009).

Posteriormente, Campbell-Stills y Stein (2007) validaron la versión reducida de esta escala mostrando una alta notoriedad debido a su brevedad y a las buenas propiedades psicométricas obtenidas. Así, Wang, Shi, Zhang y Zhang (2010) indican que el análisis factorial exploratorio realizado evidencia un único factor con buena consistencia interna $(\alpha=.91)$ y fiabilidad de test-retest $(r=.90$ para un intervalo de dos semanas). En España, Notario-Pacheco et al. (2011) aplican la escala a una muestra de 681 jóvenes universitarios y concluyen que tiene las adecuadas propiedades psicométricas para ser utilizada en población española. Serrano-Parra et al. (2012) mediante análisis factorial confirmatorio en una muestra de mayores (personas entre 60 y 75 años de edad) encuentran también un único factor subyacente y adecuada validez convergente y consistencia interna $(\alpha=.81)$. Ahora bien, pocos estudios se han interesado en demostrar sus propiedades psicométricas en trabajadores, aunque como excepción se puede citar el realizado por Lopes y Martins (2011) en una muestra de 463 trabajadores brasileños donde confirman la estructura unifactorial y un $\alpha=.82$. Así como el estudio llevado a cabo por Soler et al. (2016) en el que, además de corroborar una estructura unifactorial, se muestran las buenas propiedades psicométricas de esta escala para su utilización en trabajadores españoles $(\alpha=.87)$.

Dimensiones de la escala de resiliencia de Connor y Davidson (2003)

Los análisis de las propiedades psicométricas de la escala CD-RISC relativos a la población general muestran cinco factores: "Competencia personal, altos estándares y tenacidad", "Confianza en los propios instintos, tolerancia al afecto negativo y fortalecimiento de los efectos del estrés", "Aceptación positiva del cambio", "Control" y por último, "Espiritualidad".

Sin embargo, la estructura multifactorial de la escala de 25 ítems presenta ciertas limitaciones (Notario-Pacheco et al., 2011). Siguiendo a Campbell-Stills y Stein (2007), en primer lugar, no se establece un criterio claro para la selección de los ítems que deben de ir en cada factor. En segundo lugar, al utilizarse una rotación ortogonal en el análisis factorial exploratorio original, no se permite a los factores correlacionar. En tercer lugar, es difícil interpretar el sentido de los factores debido a que contienen ítems de temas muy diversos. Por último, el factor 5 referido a influencias espirituales está conformado por dos ítems, a pesar de que diversos metodólogos especifican que los factores deben de estar compuestos por tres o más ítems. Es por ello que Campbell-Stills y Stein (2007), tras analizar las propiedades psicométricas de la escala original sobre dos muestras distintas, proponen una versión reducida de la misma con una estructura unifactorial.

El interés de este estudio tiene su origen tanto en la escasez de estudios validación de instrumentos de medida de la resiliencia en desempleados como en la necesidad de evaluarla en este grupo de población. Por lo que se pretende analizar las propiedades psicométricas de la versión española de 10 ítems de la escala de resiliencia CD-RISC de Connor y Davidson (2003) realizada por Notario-Pacheco et al. (2011) en una muestra de desempleados.

\section{Participantes}

\section{Método}

La muestra estuvo compuesta por 226 desempleados $(42.2 \%$ hombres y $57.8 \%$ mujeres), con una media de edad de 36.54 años (SD = 8.23; rango = 19-64 años). La mayoría estaban casados o vivían en pareja (64.4\%) y el $51.6 \%$ tenían cargas familiares. Según el nivel de formación reglada, el 36.2\% poseía estudios de formación profesional, el 32.1\% universitarios, el $18.8 \%$ primarios, y el $12.9 \%$ bachillerato.

El tiempo medio en desempleo fue de 14.64 meses (SD $=3$ 2.66; rango = 0-7 años) y la media de veces que los participantes habían estado desempleados fue de $2(S D=1.63$; rango = 
0-12 veces). Atendiendo a la actividad de la última empresa, los participantes se distribuyeron en comercio (29.2\%), industria $(11.9 \%)$, construcción $(11.9 \%)$, sanidad $(9.7 \%)$, educación (8.1\%), hostelería (7\%), transporte (3.8\%) y agricultura $(2.2 \%)$. El $82 \%$ estaba trabajando en una empresa privada y el $18 \%$ en una empresa pública. La antigüedad media en la última empresa era de 3.7 años (SD = 54.91; rango $=0$ mes-27 años). En cuanto al puesto de trabajo que se desempeñaba antes de estar en situación de desempleo, el $58.1 \%$ pertenecía a la categoría de personal de base, el 36.7\% lo conformaba técnicos y el $5.1 \%$ formaba parte del grupo de directivos.

\section{Procedimiento}

La recogida de datos para la muestra se realizó en las oficinas del Servicio de Empleo y Formación (SEFCARM) de la Región de Murcia, mediante un cuestionario que incluía las instrucciones y varias escalas de auto-aplicación. Se recopilaron entre enero y marzo de 2015. Una vez completados, fueron introducidos en un sobre en blanco y cerrados por los participantes. Todos los participantes lo hicieron voluntariamente, y se garantizó el anonimato y la confidencialidad, puesto que los cuestionarios no incluyeron ningún tipo de datos personales. Se entregó una declaración de consentimiento informado en la que los participantes manifestaron su voluntad de participar en el estudio, así mismo, se puso de manifiesto los objetivos perseguidos con la realización del mencionado estudio. Se repartieron 300 cuestionarios y fueron devueltos 262, de los cuales se rechazaron 36 por no estar correctamente cumplimentados (tasa de respuesta 75.3\%).

\section{Instrumentos}

La resiliencia fue evaluada con la versión breve de la Connor-Davidson Resilience Scale (CD-RISC) en la adaptación española de Notario-Pacheco et al. (2011). Está conformada por 10 ítems (los numerados como 1, 4, 6, 7, 8, 11, 14, 16, 17, 19) de la escala original elaborada por Connor y Davidson (2003). Mediante esta escala se solicita a los participantes que respondan en qué medida están de acuerdo con cada una de las frases que se les presenta (por ejemplo, el ítem 1: "soy capaz de adaptarme a los cambios"). La forma de respuesta es una escala tipo Likert de 5 puntos, desde 0 (totalmente en desacuerdo) hasta 4 (totalmente de acuerdo). La fiabilidad de escala fue de $\alpha=.87$.

Como indicador de salud psicológica se utilizó el cuestionario GHQ-12 de Goldberg y Williams (1988) en la versión validada de Rocha, Pérez, Rodríguez-Sanz, Borrell y Obiols, (2011). Este instrumento consta de 12 ítems referidos a problemas de bienestar padecidos en las últimas semanas (por ejemplo: "¿se ha sentido constantemente agobiado y en tensión?", ítem 5). Su evaluación se realiza mediante una escala tipo Likert de 4 puntos desde 1 (no, en absoluto) hasta 4 (mucho más de lo habitual), por tanto, altas puntuaciones indican peor nivel de salud. La fiabilidad de escala fue de $\alpha=.91$.

Para la valoración y medida de la sintomatología psicosomática se utilizó la escala adaptada de Hock (1988) en su validación al español por García-Izquierdo, Castellón, Albadalejo y García-Izquierdo (1993). Consta de 12 ítems con un rango de respuesta de 0 a 6 , desde "nunca" hasta "con mucha frecuencia", referidos a los síntomas o molestias ("jaquecas y dolores", "sensación de cansancio", "disminución del apetito") que ha padecido el encuestado durante los últimos tres meses. La fiabilidad de escala fue de $\alpha=.88$.

Y por último, la escala de Autoeficacia General de Baessler y Schwarzer (1996) que evalúa el sentimiento estable de competencia personal para manejar de forma eficaz una gran variedad de situaciones estresantes. En su versión española de Suarez, Gracia y Moreno (2000), cuenta con un total de 10 ítems (por ejemplo: "venga lo que venga, por lo general soy capaz de manejarlo", ítem número 7). El formato de respuesta es de tipo Likert con cuatro opciones de respuesta, desde 1 (totalmente en desacuerdo) hasta 4 (totalmente de acuerdo). La fiabilidad de escala fue de $\alpha=.89$.

\section{Análisis de datos}

En primer lugar, se realizaron los estadísticos descriptivos y análisis de fiabilidad de las escalas con el programa SPSS 21.0. En segundo lugar, mediante el programa EQS 6.1, se llevó a cabo un Análisis Factorial Confirmatorio (AFC) con el método de estimación de máxima verosimilitud.

Por último, con el programa SPSS 21.0 se analizó la validez convergente, divergente y de criterio. En este último caso, mediante las correlaciones entre las puntuaciones obtenidas en el CD-RISC y las puntuaciones de las escalas de autoeficacia, salud psicológica y síntomas psicosomáticos.

\section{Resultados}

Se realizó un análisis factorial confirmatorio (AFC) con las puntuaciones de la escala CD-RISC, ya que otros estudios previos ya habían justificado su unidimensionalidad (Lopes \& Martins, 2011; Serrano-Parra et al., 2012; Soler et al., 2016; Wang et al., 2010). Se utilizó el método de estimación de máxima verosimilitud para explorar las relaciones entre las variables. La bondad de ajuste del modelo propuesto se evaluó mediante diversos indicadores: $\chi 2$ dividido por los grados de libertad, el promedio de los residuales (RMR), el promedio de los residuales estandarizados (RMSEA), el índice de bondad de ajuste GFI y el índice de ajuste comparativo CFI. Para que exista un buen ajuste, los valores GFI y CFI deben superar el valor .90, los valores RMSEA deben estar cercanos a .05, y el coeficiente $\chi 2 / g l$. ha de ser menor o igual a 3. Los resultados del AFC (que se pueden observar en la Tabla 1 y en la figura 1 la representación gráfica del modelo) corroboran el modelo unifactorial. 
Tabla 1. Índices de bondad de ajuste del modelo de un factor del CD-RISC 10.

\begin{tabular}{llllll}
\hline Modelo factorial & $\chi 2 / g l$ & CFI & GFI & RMR & .95 \\
\hline Un factor & .00002 & .96 & .94 & .05 \\
Adecuación & Buena & Buena & Buena & Buena & Buena \\
\hline
\end{tabular}

Para analizar la validez convergente se obtuvieron las correlaciones entre las puntuaciones de la escala CD-RISC y las obtenidas en las escalas de autoeficacia, salud psicológica y síntomas psicosomáticos. En la Tabla 2, pueden observarse

Tabla 2. Estadísticos descriptivos y correlaciones de las variables principales del estudio. los estadísticos descriptivos y las correlaciones entre las variables. Todas las correlaciones son estadísticamente significativas y tienen el sentido teórico esperado.

\begin{tabular}{|c|c|c|c|c|c|c|}
\hline & $M$ & $S d$ & 1 & 2 & 3 & 4 \\
\hline 1. Resiliencia & 3.15 & .70 & $(.87)$ & & & \\
\hline 2. Autoeficacia & 3.18 & .50 & $.64^{* *}$ & $(.89)$ & & \\
\hline 3. Síntomas psicosomáticos & 2.36 & .92 & $-.36 * *$ & $-.28^{* *}$ & $(.88)$ & \\
\hline 4. Salud & 1.91 & .54 & $-.49 * *$ & $-.36 * *$ & $.69 * *$ & $(.91)$ \\
\hline
\end{tabular}

${ }^{* *} \mathrm{p}<.01 . \mathrm{N}=226$. Nota: Entre paréntesis se muestran los coeficientes de fiabilidad de las escalas.

\section{Discusión}

El objetivo de este estudio ha sido examinar las propiedades psicométricas de la medida de la resiliencia CD-RISC de 10 ítems en una muestra de desempleados. De acuerdo con los datos obtenidos, esta escala posee unas adecuadas propiedades psicométricas.

En este caso, el modelo de un único factor muestra adecuados índices de bondad, como ocurre con los estudios llevados a cabo por Campbell-Stills y Stein (2007), Wang et al. (2010), Lopes y Martins, (2011), Hartley (2012) y, en España, Notario-Pacheco et al. (2011), Serrano-Parra et al. (2012) y Soler et al. (2016) sobre población general, estudiantes y trabajadores.

En cuanto a la consistencia interna, en el presente estudio se halló un $\alpha=.87$, algo superior a los expuestos en los estudios de Campbell-Stills y Stein $(2007, \alpha=.85)$, Notario-Pacheco et al. $(2011, \alpha=.85)$ y Lopes y Martins $(2011, \alpha=.82)$, pero inferior al obtenido por Wang et al. (2010, $\alpha=.91)$.

Para analizar la validez convergente, se optó por utilizar variables que en el contexto del desempleo pueden tener una gran repercusión, como son la salud psicológica, los síntomas psicosomáticos y la autoeficacia. Las relaciones entre las puntuaciones de la escala de resiliencia y las de las variables criterio han sido estadísticamente significativas en todos los casos y en la dirección teórica esperada. Numerosos estudios han mostrado el efecto negativo que la situación de desempleo provoca en la salud de los desempleados (Ford et al., 2010; McKee-Ryan et al., 2005). Por otro lado, en relación a la importancia que tienen los recursos personales a la hora de enfrentarse a la situación de desempleo (Fugate, Kinicki \& Ashfor, 2004; Thijsen, Van der Heijden \& Rocco, 2008), la autoeficacia se ha mostrado como posible amortiguador de los efectos del desempleo sobre el bienestar de los desempleados (Vinokur \& Schul, 2002) así como un posible indicador de la eficacia de las conductas de búsqueda de empleo (Saks \& Ashforth, 1999; Taggar \& Kuron, 2016).

Es necesario poner de relieve la importancia de contar con un instrumento de medida de la resiliencia para la población desempleada. Como se ha indicado antes, la resiliencia es una variable poco estudiada en este campo, a pesar de que puede ser muy beneficiosa cuando las personas deben de afron- 
tar procesos estresantes (Baek et al., 2010; Jackson, Firtko \& Edenborough, 2007), sin olvidar las relaciones de esta variable con la salud y con las conductas de búsqueda de empleo (Dimas, Pereira \& Canavarro, 2013; Moorhouse \& Caltabiano, 2007; Sweet \& Mohen, 2012).

Con este estudio se pone a disposición un instrumento de medida de la resiliencia válido y fiable para muestras de desempleados españoles según las evidencias estadísticas presentadas, por lo que se espera que se profundice más en el análisis de esta variable en contextos de búsqueda de empleo. El hecho de poder evaluar la resiliencia en este ámbito es útil en dos vertientes: por un lado, permite profundizar en el papel de este recurso psicológico a la hora de analizar la influencia del mismo tanto en la inserción laboral de las personas en situación de desempleo como sobre su estado de salud. Por otro lado, la resiliencia es considerada como una variable más cercana a los estados que a los rasgos y, por tanto, es susceptible de ser maleable (Luthans, Youssef-Morgan \& Avolio, 2015). Ello permite la propuesta de programas de intervención dirigidos a desempleados para mejorar el desarrollo de este recurso psicológico. Ya en el año 2006, Luthans, Avey, Avolio, Norman y Combs, proponen un programa de formación en capital psicológico en el que una de las sesiones estaba dedicada a la resiliencia, y que si bien no se realizó en población desempleada, queda patente la eficacia de este tipo de intervenciones para promover el desarrollo de variables como la resiliencia. En referencia a las tendencias actuales de intervención en desempleados, es destacable tanto la línea de investigación basada en el desarrollo de competencias socioemocionales (Pérez \& Ribera, 2009) como la relativa a la aplicación de la psicología positiva al ámbito del desempleo (Hodzic, Ripoll, Lira \& Zenasni, 2015). En ambas se podría encuadrar una intervención basada en el desarrollo de la resiliencia en población desempleada. Por tanto, la evaluación de este recurso personal facilita el análisis de la eficacia de las intervenciones y programas de formación dirigidos a desempleados basados en su desarrollo.

El estudio contiene algunas limitaciones que conviene señalar. En primer lugar, los datos han sido recogidos mediante auto-informe. Esta es una práctica muy habitual en múltiples investigaciones que puede dar lugar a un sesgo en la respuesta de los participantes, exacerbar la varianza común y aumentar artificialmente las correlaciones entre variables (Spector, 2006).

En segundo lugar, la muestra está compuesta por desempleados españoles que tienen sus propias características culturales; por lo tanto, los resultados obtenidos no pueden extrapolarse a otras muestras. Sería interesante la realización de estudios interculturales o transnacionales para verificar si los resultados de nuestro trabajo son semejantes a los de otros países.

En tercer lugar, hemos utilizado un diseño transversal que no permite poner a prueba relaciones causales. Lo que invita a investigaciones futuras a verificar las relaciones entre variables mediante un diseño longitudinal.

En cuarto lugar, es importante resaltar que la resiliencia es un concepto multidimensional que puede verse afectado por otros factores (biológicos o contextuales) no tenidos en cuenta en la presente investigación.
Por último, en referencia a las líneas futuras de investigación y teniendo en cuenta que la resiliencia se entiende como un proceso dinámico, sería beneficioso para el desarrollo del concepto que, en futuras investigaciones, se tuviesen en cuenta diseños longitudinales que pudieran poner de relieve la naturaleza dinámica de la misma, sobre todo, en población desempleada.

\section{Agradecimientos}

Consejería de Industria, Turismo, Empresa e Innovación de la CARM, a través de la Fundación Séneca, Agencia de Ciencia y Tecnología de la Región de Murcia, por medio de una ayuda del Programa Séneca "Contratos Predoctorales de Formación del Personal Investigador" otorgado a la primera autora. 


\section{Referencias}

Ahern, N., Kiehl, E., Sole, M. \& Byers, J. (2006). A Review of Instruments Measuring Resilience. Issues in Comprehensive Pediatric Nursing, 29(2), 103-125. doi:10.1080/01460860600677643

Baek, H., Lee, K., Joo, E., Lee, M. \& Choi, K. (2010). Reliability and Validity of the Korean Version of the Connor-Davidson Resilience Scale. Psychiatry Investigation, 7(2), 109-121. doi:10.4306/pi.2010.7.2.109

Baessler, J. \& Schwarzer, R. (1996). Evaluación de la autoeficacia: Adaptación española de la escala de Autoeficacia General. Ansiedad y Estrés, 2(1), 1-8.

Baruth, K. \& Carroll, J. (2002). A formal assessment of resilience: The Baruth Protective Factors Inventory. Journal of Individual Psychology, 58(3), 235-244.

Becoña, E. (2006). Resiliencia: definición, características y utilidad del concepto. Revista de Psicopatología y Psicología Clínica, 11(3), 125-145.

doi:10.5944/rppc.vol.11.num.3.2006.4024

Beland, F., Birch, S. \& Stoddart, G. (2002). Unemployment and health: contextual-level influences on the production of health in populations. Social Science \& Medicine, 55(11), 2033-2052. doi:10.1016/S02779536(01)00344-6

Burgard, S. A., Brand, J. E., \& House, J. S. (2007). Toward a Better Estimation of the Effect of Job Loss on Health. Journal of Health and Social Behavior, 48(4), 369384. doi:10.1177/002214650704800403

Campbell-Stills, L. \& Stein, M. (2007). Psychometric Analysis and Refinement of the Connor-Davidson Resilience Scale (CD-RISC): Validation of a 10-Item Measure of Resilience. Journal of Traumatic Stress, 20(6), 1019-1028. doi:10.1002/jts.20271

Connor, K. \& Davidson, J. (2003). Development of a new resilience scale: the Connor-Davidson Resilience Scale (CD-RISC). Depress and Anxiety, 18(2), 76-82. doi:10.1002/da.10113

Csikszentmihalyi, M. (1999). If we are so rich, why aren't we happy? American Psychologist, 54(10), 821-827. doi:10.1037\%2F0003-066X.54.10.821

Del Pozo, J., Ruiz, M., Pardo, A. \& San Martín, R. (2002). Efectos de la duración del desempleo entre los desempleados. Psicothema, 14(2), 440-443. Recuperado de http://www.psicothema.com/psicothema.asp?id $=745$

Dimas, I., Pereira, M. \& Canavarro, M. (2013). Ajustamento psicossocial, ajustamento diádico e resiliência no contexto de desemprego. Análise Psicológica, 31(1), 3-16. Recuperado de http://www. scielo.mec.pt/scielo.php?script=sci_arttext\&pi$\mathrm{d}=$ S0870-82312013000100001

Fergus, S. \& Zimmerman, M. (2005). Adolescent resilience: A framework for understanding healthy development in the face of risk. Annual Review of Public Health, 26(1), 399-419. doi:10.1146/annurev.publhealth.26.021304.144357

Fernández-Lansas, V. \& Crespo L. (2011). Resiliencia, Personalidad Resistente y Crecimiento en Cuidadores de Personas con Demencia en el Entorno Familiar: Una Revisión. Clínica y Salud, 22(1), 21-40. doi:10.5093/cl2011v22n1a2

Ferrie, J., Shipley, M., Marmot, M., Martikainen, P., Stansfeld, S. \& Smith, G. (2001). Job insecurity in white-co- llar workers: Toward an explanation of association with health. Journal of Occupational Health Psychology, 6(1), 26-42. doi:10.1037/1076-8998.6.1.26

Ford, E., Clark, C., McManus, S., Harris, J., Jenkins, R., Bebbington, P. \& Stansfeld, S. (2010). Common mental disorders, unemployment and welfare benefits in England. Public Health, 124(12), 675-681. doi:10.1016/j.puhe.2010.08.019

Fugate, M., Kinicki, A. \& Ashforth, B. (2004). Employability: A psycho-social construct, its dimensions, and applications. Journal of Vocational Behavior, 65(1), 14-38. doi:10.1016/j.jvb.2003.10.005

García-Izquierdo, M., Castellón, M., Albadalejo, B. \& García-lzquierdo, A. (1993). Relaciones entre Burnout, ambigüedad de rol y satisfacción laboral en el personal de Banca. Revista de Psicología del Trabajo y de las Organizaciones, 9(24), 17-26. Recuperado de http://www.copmadrid.org/webcopm/publicaciones/trabajo/1993/vol1/arti2.htm

García-Izquierdo, A., Ramos-Villagrasa, P. \& García-Izquierdo, M. (2009). Los Big Five y el efecto moderador de la resistencia en el agotamiento emocional. Revista de Psicología del Trabajo y las Organizaciones, 25(2), 135-147. Recuperado de http://www.redalyc. org/pdf/2313/231316498004.pdf

Gillespie, B., Chaboyer, W. \& Wallis, M. (2009). The influence of personal characteristics on the resilience of operating room nurses: A predictor study. International Journal of Nursing Studies, 46(7), 968-976. doi:10.1016/j.ijnurstu.2007.08.006

Goldberg, D. \& Williams, P. (1988). A user's guide to the General Health Questionnaire. Windsor: Nfer-Nelson.

González-Arratia, N., Valdez, J. \& González, S. (2011). Investigación en resiliencia: ¿Qué hemos aprendido? En J. Moral de la Rubia, L. Valdez \& N. González-Arratia (Eds.), Psicología y Salud (pp. 157-172). Coahuila, México: Consorcio de Universidades Mexicanas.

Grotberg, E. (1995). A guide to promoting resilience in children: strengthening the human spirit. The Hague, The Netherlands: Bernard Van Leer Foundation.

Grotberg, E. (2002). From terror to triumph: The path to resilience. In C. Stout (Ed.), The psychology of terrorism (pp. 167-189). Westport, CT: Praeger.

Guarino, L. \& Sojo, V. (2011). Apoyo social como moderador del estrés en la salud de los desempleados. Universitas Psychologica, 10(3), 867-879.

Hanish, K.A. (1999), Job loss and unemployment research from 1994 to 1998: A review and recommendations for research and intervention. Journal of Vocational Behavior, 55,188-220.doi:10.1006/jvbe.1999.1722

Hartley, M. (2012). Assessing and promoting resilience: An additional tool to address the increasing number of college students with psychological problems. Journal of College Counseling, 15(1), 37-51. doi:10.1002/j.2161-1882.2012.00004.x

Herrman, H., Stewart, D., Díaz-Granados, N., Berger, E., Jackson, B. \& Yuen, T. (2011). What is resilience? The Canadian Journal of Psychiatry, 56(5), 258-265. doi:10.1177/070674371105600504

Hock, R. (1988). Professional burnout among public school teachers. Public Personnel Management, 17(2), 167189. doi:10.1177/009102608801700207

Hodzic, S., Ripoll, P., Lira, E. \& Zenasni, F. (2015). Can intervention in emotional competences increase em- 
ployability prospects of unemployed adults? Journal of Vocational Behavior, 88, 28-37. doi:10.1016/j. jvb.2015.02.007

Jackson, D., Firtko, A. \& Edenborough, M. (2007). Personal resilience as a strategy for surviving and thriving in the face of workplace adversity: a literature review. Journal of Advanced Nursing, 60(1), 1-9. doi:10.1111/ j.1365-2648.2007.04412.x

Lamond, A., Depp, C., Allison, M., Langer, R., Reichstadt, J., Moore, D., ... \& Jeste, D. (2008). Measurement and predictors of resilience among community-dwelling older women. Journal of Psychiatric Research, 43(2), 148-154. doi:10.1016/j.jpsychires.2008.03.007

Lim, V., Chen, D. \& Tan, M. (2013). Unemployed and Exhausted? Fatigue During Job-Search and Its Impact on Reemployment Quality. Academy of Management Proceedings, 92(1), 68-78. doi:10.5465/ambpp.2013.13399abstract

Liu, D., Fairweather-Schmidt, A., Burns, R. \& Roberts, R. (2015). The Connor-Davidson Resilience Scale: establishing invariance between gender across the lifespan in a large community-based cohort. Journal of Psychopathology and Behavior Assessment, 37(2), 109-132. doi:10.1007/s10862-014-9452-z

Lopes, V. \& Martins, M. (2011). Validação fatorial da escala de resiliência de connor-davidson (CD-RISC-10) para brasileiros. Revista Psicologia, 11(2), 36-50. Recuperado de http://pepsic.bvsalud.org/pdf/rpot/ v11n2/v11n2a04.pdf

Luthans, F. (2002). The need for and meaning of positive organizational behavior. Journal of Organizational Behavior, 23(6), 695-706. doi:10.1002/job.165

Luthans, F., Avey, J. B., Avolio, B. J., Norman, S. M., \& Combs, G. M. (2006). Psychological capital development: toward a micro-intervention. Journal of Organizational Behavior, 27(3), 387-393. doi:10.1002/job.373

Luthans, F., Vogelgesang, G. \& Lester, P. (2006). Developing the psychological capital of resiliency. $\mathrm{Hu}$ man Resource Development Review, 5(1), 25-44. doi:10.1177/1534484305285335

Luthans, F., Youssef-Morgan, C. \& Avolio, B. (2015). Psychological capital and beyond. Oxford University Press, USA.

Luthar, S. \& Becker, B. (2002). Privileged but Pressured? A Study of Affluent Youth. Child Development, 73(5), 1593-1610. doi:10.1111/1467-8624.00492

Luthar, S., Cicchetti, D. \& Becker, B. (2000). The construct of resilience: A critical evaluation and guidelines for future work. Child Development, 71(3), 543-562. doi:10.1111/1467-8624.00164

Masten, A., Cutuli, J., Herbers, J. \& Reed, M. (2009). Resilience in development. In C. Snyder \& S. Lopez (Eds.), Oxford Handbook of Positive Psychology (2nd ed., pp. 117-131). New York: Oxford University Press.

Masten, A. \& Reed, M. (2002). Resilience in development. In C. Snyder \& S. Lopez (Eds.), The handbook of positive psychology (pp. 74-88). New York: Oxford University Press.

McKee-Ryan, F., Song, Z., Wanberg, C. \& Kinicki, A. (2005). Psychological and physical well-being during unemployment: a meta-analytic study. Journal of Applied Psychology, 90(1), 53-76. doi:10.1037/00219010.90.1.53

Méndez, I., García, J., Martínez, J., Boti, M., Cánovas, A. \& Cle- mente, Y. (2015). Resiliencia en trabajadores y en residentes de un centro de personas mayores institucionalizadas. European Journal of Investigation in Health, Psychology and Education, 5, 65-73. doi:10.1989/ejihpe.v1i1.91

Mol, S., Arntz, A., Metsemakers, J., Dinant, G., Montfort, P., Vilters-Van, A. \& Knottnerus, J. (2005). Symptoms of post-traumatic stress disorder after non-traumatic events: Evidence from an open population study.British Journal of Psychiatry, 186, 494-499. doi:10.1192/bjp.186.6.494

Moorhouse, A. \& Caltabiano, M. (2007). Resilience and unemployment: Exploring risk and protective influences for the outcome variables of depression and assertive job searching. Journal of Employment Counseling, 44(3), 115-125. doi:10.1002/j.2161-1920.2007. tb00030.x

Moyano-Díaz, E., Gutiérrez, D., Zúñiga, K. \& Cornejo-Troncoso, F. (2013). Empleados y desempleados, atribuciones causales y bienestar subjetivo. Psicologia \& Sociedade, 25(2), 440-450. doi:10.1590/s010271822013000200021

Notario-Pacheco, B., Solera, M., Serrano, M., Bartolomé, R., García-Campayo, J. \& Martínez-Vizcaíno, V. (2011). Reliability and validity of the Spanish version of the 10 item Connor -Davidson Resilience Scale (10 item CDRISC) in young adults. Health and Quaity of Life Outcomes, 9(1), 63-81. doi:10.1186/14777525-9-63

Paul, K. \& Moser, K. (2009). Unemployment impairs mental health: Meta-analyses. Journal of Vocational Behavior, 74(3), 264-282. doi:10.1016/j.jvb.2009.01.001

Pérez, N. \& Ribera, A. (2009). Las competencias emocionales en los procesos de inserción laboral. Revista Española de Orientación y Psicopedagogía, 20(3), 251256. Recuperado de http://diposit.ub.edu/dspace/ bitstream/2445/50903/1/575400.pdf

Pipe, T., Buchda, V., Launder, S., Hudak, B., Hulvey, L., Karns, K. \& Pendergast, D. (2012). Building personal and professional resources of resilience and agility in the healthcare workplace. Stress and Health, 28(1), 11-22. doi:10.1002/smi.1396

Richardson, G. (2002). The metatheory of resilience and resiliency. Journal of Clinical Psychology, 58(3), 307321. doi:10.1002/jclp.10020

Rocha, K., Pérez, K., Rodríguez-Sanz, M., Borrell, C. \& Obiols, J. (2011). Propiedades psicométricas y valores normativos del General Health Questionnaire (GHQ12) en población general española. International Journal of Clinical and Health Psychology, 11(1), 125-139. Recuperado de http://www.redalyc.org/ articulo.oa?id=33715423008

Rutter, M. (1993). Resilience: Some conceptual considerations. Journal of Adolescent Health, 14(8), 626-631. doi:10.1016/1054-139x(93)90196-v

Saks, A. \& Ashforth, B. (1999). Effects of individual differences and job search behaviors on the employment status of recent university graduates. Journal of Vocational Behavior, 54(2), 335-349. doi:10.1006/ jvbe.1998.1665

Serrano-Parra, M., Garrido, M., Notario-Pacheco, B., Bartolomé, R., Solera, M. \& Martínez, V. (2012). Validez de la escala de Resiliencia de Connor-Davidson (CD-RISC) en una población de mayores entre 60 
y 75 años. International Journal of Psychological Research, 5(2), 49-57. doi:10.21500/20112084.736

Ślebarska, K., Moser, K. \& Gunnesch-Luca, G. (2009). Unemployment, social support, individual resources, and job search behavior. Journal of Employment Counseling, 46(4), 159-170. doi:10.1002/j.2161-1920.2009. tb00079.x

Soler, M., Meseguer, M. \& García-Izquierdo, M. (2016). Propiedades psicométricas de la versión española de la escala de resiliencia de 10 ítems de Connor-Davidson (CD-RISC 10) en una muestra multiocupacional. Revista Latinoamericana de Psicología, 48(3), 159-166. doi:10.1016/j.rlp.2015.09.002

Spector, P. (2006). Method variance in organizational research truth or urban legend?. Organizational Research Methods, 9(2), 221-232. doi:10.1177/1094428105284955

Suárez, P. S., García, A. M. P., \& Moreno, J. B. (2000). Escala de autoeficacia general: datos psicométricos de la adaptación para población española. Psicothema, 12(Suplemento), 509-513.

Sutherland, J., Cook, L., Stetina, P. \& Hernández, C. (2009). Women in substance abuse recovery: measures of resilience and self-differentiation. Western Journal of Nursing Research, 31(7), 905-922. doi:10.1177/0193945909337815

Sweet, S. \& Moen, P. (2012). Dual earners preparing for job loss: Agency, linked lives, and resilience. Work and Occupations, 39(1), 35-70. doi:10.1177/0730888411415601

Taggar, S. \& Kuron, L. (2016). The toll of perceived injustice on job search self-efficacy and behavior. Career Development International, 21(3), 279-298. doi:10.1108/ cdi-10-2015-0139

Taormina, R. (2015). Adult Personal Resilience: A New Theory, New Measure, and Practical Implications. Psychological Thought, 8(1), 35-46. doi:10.5964/psyct. v8i1.126

Thijssen, J., Van der Heijden, B. \& Rocco, T. (2008). Toward the employability-link model: current employment transition to future employment perspectives. $\mathrm{Hu}$ man Resource Development Review, 7(2), 165-183. doi:10.1177/1534484308314955

Vansteenkiste, V., Lens, W., De Witte, H. \& Feather, N. (2005). Understanding unemployed people's job search behaviour, unemployment experience and well-being: A comparison of expectancy-value theory and self-determination theory. British Journal of Social Psychology, 44(2), 269-287. doi:10.1348/014466604x17641

Vinokur, A. \& Schul, Y. (2002). The web of coping resources and pathways to reemployment following a job loss. Journal of Occupational Health Psychology, 7(1), 68-83. doi:10.1037/1076-8998.7.1.68

Wagnild, G. \& Young, H. (1993). Development and psychometric properties of a resilience scale. Journal of Nursing Measurement, 1(2), 165-178. doi:10.1037/ t07521-000

Wang, L., Shi, Z., Zhang, Y. \& Zhang, Z. (2010). Psychometric properties of the 10-item Connor-Davidson Resilience Scale in Chinese earthquake victims. Psychiatry and Clinical Neurosciences. 64(5), 499504. doi:10.1111/j.1440-1819.2010.02130.x

Windle, G., Bennett, K. \& Noyes, J. (2011). A methodological review of resilience measurement scales. Health and Quality of Life Outcomes, 9(1), 8. doi:10.1186/14777525-9-8 